J. Gynäkol. Endokrinol. AT 2021 · 31:39-40 https://doi.org/10.1007/s41974-021-00173-z Angenommen: 11. Januar 2021

๑ Der/die Autor(en) 2021

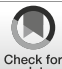

\section{Klara Beitl}

Abteilung für Gynäkologische Endokrinologie und Reproduktionsmedizin, Universitätsklinik für Frauenheilkunde, Wien, Österreich

\title{
Primäre Ovarialinsuffizienz - Bedeutung der Autoimmunität und verminderten Knochendichte
}

Im Rahmen des Klimakteriums kommt es in physiologischer Weise zum Erliegen der ovariellen Funktion. Im Durchschnitt tritt dieser Zustand mit 52 Jahren ein. Als primäre Ovarialinsuffizienz (POI) wird ein Krankheitsbild bezeichnet, bei dem es bereits vor dem 40. Lebensjahr zu hormonellen Veränderungen im Sinne eines hypergonadotropen Hypogonadismus kommt. Die Symptome entsprechen jenen der Menopause. Definitionsgemäß spricht man von POI bei FSH-Spiegeln > 25 IU/l in Kombination mit einer über 4 Monate anhaltenden Oligo- bzw. Amenorrhö [1]. Die Prävalenz vor dem 40 . Lebensjahr liegt bei $1-2 \%$, vor dem 30. Lebensjahr bei $0,1 \%[2,3]$.

Bei bestehendem Östrogendefizit leiden betroffene Patientinnen oftmals unter Hitzewallungen, Schlafstörungen, vaginaler Atrophie und damit einhergehender vaginaler Trockenheit. Darüber hinaus kommt es durch den Mangel an Sexualhormonen in den meisten Fällen zu einer Anovulation und folglich zu einer Infertilität. Nichtsdestotrotz haben viele POI-Patientinnen eine intermittierende ovarielle Funktion mit Wiederkehr einer Ovulation und Menstruation. Bei Patientinnen mit unregelmäßigen Zyklen darf daher nicht irrtümlich die Diagnose einer POI verfehlt werden [4].

Ist die Diagnose POI gestellt, so bedeutet dies für viele Frauen eine starke emotionale Belastung. Bei Kinderwunsch und Familienplanung sollte auf die 5-10\% hohe Chance einer Schwangerschaft hingewiesen werden [5].
Einen wichtigen Stellenwert bei POI nehmen die Knochendichte und kardiovaskuläre Gesundheit ein. Das lang anhaltende Östrogendefizit bei diesen Patientinnen zeigt sowohl einen negativen Einfluss auf die Knochendichte als auch auf die kardiovaskuläre Morbidität und Mortalität. Bisherige Daten zeigen bei ca. zwei Drittel aller POI-Patientinnen eine erniedrigte Knochendichte [6, 7]. Die Prävalenz ist steigend mit dem Alter und korreliert ebenso mit der Dauer der bestehenden Amenorrhö und damit der Länge des Östrogendefizits [7, 8]. Eine frühe Diagnosestellung ist vor allem in Hinblick auf ein zunehmendes Alter und den Zeitpunkt der „peak bone mass" um das 30. Lebensjahr von großer Bedeutung, um möglichst früh eine Hormonersatztherapie einleiten zu können.

In $90 \%$ der Fälle ist die Ätiologie der primären Ovarialinsuffizienz ungeklärt. Die Krankheitsentstehung tritt beispielweise im Rahmen einer vorhergehenden Operation, Radio- bzw. Chemotherapie auf, kann aber ebenso genetisch oder infektiös bedingt sein. Darüber hinaus sind autoimmune Zusammenhänge beschrieben [9]. Es wird geschätzt, dass bei etwa $10-40 \%$ der Patientinnen mit POI eine Autoimmunerkrankung vorliegt $[9,10]$. Die am häufigsten assoziierten Erkrankungen sind autoimmune Schilddrüsenerkrankungen (27\%), Morbus Addison (2,5\%) und Diabetes mellitus Typ 1 (2,5\%; [11-13]).

In vorhergehenden Studien wurde ein möglicher Zusammenhang zwischen Autoimmunität und Knochendichte be- schrieben. Es hat sich gezeigt, dass Antikörper einen Einfluss auf die Osteoklastendifferenzierung und -aktivierung haben können und somit zu einer Veränderung des Knochenmineralgehalts beitragen [14]. Was diese Annahme unterstützt, sind einige Studien, die eine verminderte Knochendichte bei Autoimmunerkrankungen wie systemischem Lupus erythematodes, chronischer Thyreoiditis und autoimmuner Hepatitis gezeigt haben [15-17]. Ein möglicher Zusammenhang zwischen Knochendichte und Autoimmunität bei POIPatientinnen ist jedoch bisher kaum untersucht.

In einer kürzlich publizierten Studie von Beitl et al. 2020 wurde in einem POI-Kollektiv von 58 Patientinnen bei rund $60 \%$ eine erniedrigte Knochendichte gefunden, der Großteil davon $(48,3 \%)$ zeigte Werte im osteopenischen Bereich. Bei ca. $36 \%$ zeigten sich autoimmune Auffälligkeiten. Bereits in der Literatur ist ein Zusammenhang zwischen Autoimmunität und erniedrigter Knochendichte beschrieben. Auch in der zuvor angeführten Studie konnte ein signifikant negativer Einfluss der Autoimmunität auf die Knochendichte bei POI-Patientinnen gezeigt werden [7]. Die Autoimmunität nimmt daher im Rahmen der POI sicherlich einen besonderen Stellenwert ein. Hinsichtlich Autoimmunerkrankungen gibt es bis dato bei POI-Patientinnen noch keine einheitlichen Screening-Guidelines. Nach Empfehlung der ESHRE Group soll bei unklarer Ätiologie oder Verdacht 
auf Autoimmunerkrankung nach TPOAntikörpern und 21-OH-Antikörpern gesucht werden [1].

In Zusammenfassung ist bei Patientinnen mit POI eine frühe Diagnosestellung zur Einleitung einer Hormonersatztherapie vor allem in Hinblick auf die Knochendichte von enormer Bedeutung. Nach Diagnosestellung ist ein umfassendes Management zu gewährleisten, welches Infertilität und Knochengesundheit, aber ebenso das kardiovaskuläre, sexuelle und emotionale Wohlergehen umfasst.

\section{Korrespondenzadresse}

\section{Dr. Klara Beit}

Abteilung für Gynäkologische Endokrinologie und Reproduktionsmedizin, Universitätsklinik für Frauenheilkunde

Währinger Gürtel 18-20, 1090 Wien, Österreich klara.beitl@meduniwien.ac.at

Funding. Open access funding provided by Medical University of Vienna.

\section{Einhaltung ethischer Richtlinien}

Interessenkonflikt. K. Beitl gibt an, dass kein Interessenkonflikt besteht.

Für diesen Beitrag wurden von der Autorin keine Studien an Menschen oder Tieren durchgeführt. Für die aufgeführten Studien gelten die jeweils dort angegebenen ethischen Richtlinien.

Open Access. Dieser Artikel wird unter der Creative Commons Namensnennung 4.0 International Lizenz veröffentlicht, welche die Nutzung, Vervielfältigung Bearbeitung, Verbreitung und Wiedergabe in jeglichem Medium und Format erlaubt, sofern Sie den/die ursprünglichen Autor(en) und die Quelle ordnungsgemäß nennen, einen Link zur Creative Commons Lizenz beifügen und angeben, ob Änderungen vorgenommen wurden.

Die in diesem Artikel enthaltenen Bilder und sonstiges Drittmaterial unterliegen ebenfalls der genannten Creative Commons Lizenz, sofern sich aus der Abbildungslegende nichts anderes ergibt. Sofern das betreffende Material nicht unter der genannten Creative Commons Lizenz steht und die betreffende Handlung nicht nach gesetzlichen Vorschriften erlaubt ist, ist für die oben aufgeführten Weiterverwendungen des Materials die Einwilligung des jeweiligen Rechteinhabers einzuholen.

Weitere Details zur Lizenz entnehmen Sie bitte de Lizenzinformation auf http://creativecommons.org/ licenses/by/4.0/deed.de.

\section{Literatur}

1. Webber L, Davies M, Anderson R, Bartlett J, Braat D, Cartwright B, Cifkova R, de Muinck Keizer-Schrama S, Hogervorst E, Janse F, Liao L, Vlaisavljevic V, Zillikens C, Vermeulen N (2016) ESHRE Guideline: management of women with premature ovarian insufficiency. Hum Reprod 31(5):926-937. https://doi.org/10.1093/humrep/ dew027

2. Podfigurna-Stopa A, Czyzyk A, Grymowicz M, Smolarczyk R, Katulski K, Czajkowski K, Meczekalski B (2016) Premature ovarian insufficiency: the context of long-term effects. J Endocrinol Invest 39(9):983-990. https://doi.org/10.1007/s40618 016-0467-z

3. Coulam CB, Adamson SC, Annegers JF (1986) Incidence of premature ovarian failure Obstet Gynecol Clin North Am 67(4):604-606

4. Welt CKM (2020) Clinical manifestations and diagnosis of spontaneous primary ovarian insufficiency (premature ovarian failure). UpToDate. https://www.uptodate.com/contents/clinicalmanifestations-and-diagnosis-of-spontaneousprimary-ovarian-insufficiency-prematureovarian-failure.Zugegriffen: 20. Okt. 2020

5. van Kasteren YM, Schoemaker J (1999) Premature ovarian failure: a systematic review on therapeutic interventions to restore ovarian function and achieve pregnancy. Hum Reprod Update 5(5):483-492. https://doi.org/10.1093/humupd/ 5.5 .483

6. Anasti JN, Kalantaridou SN, Kimzey LM, Defensor RA, Nelson LM (1998) Bone loss in young women with karyotypically normal spontaneous premature ovarian failure. Obstet Gynecol Clin North Am 91(1):12-15. https://doi.org/10.1016/ s0029-7844(97)00583-8

7. Beitl K, Rosta K, Poetsch N, Seifried M, Mayrhofer D, Soliman B, Marculescu R, Ott J (2020) Autoimmunological serum parameters and bone mass density in premature ovarian insufficiency: a retrospective cohort study. Arch Gynecol Obstet. https://doi.org/10.1007/s00404-020-05860-4

8. Popat VB, Calis KA, Vanderhoof VH, Cizza G, Reynolds JC, Sebring N, Troendle JF, Nelson LM (2009) Bone mineral density in estrogen-deficient young women. J Clin Endocrinol Metab 94(7):2277-2283. https://doi.org/10.1210/jc. 2008-1878

9. Sharif K, Watad A, Bridgewood C, Kanduc D, Amital H, Shoenfeld Y (2019) Insights into the autoimmune aspect of premature ovarian insufficiency. Best Pract Res Clin Endocrinol Metab 33(6):101323. https://doi.org/10.1016/j.beem. 2019.101323

10. Zhen X, Qiao J, Li R, Wang L, Liu P (2014) Serologic autoimmunologic parameters in women with primary ovarian insufficiency. BMC Immunol 15:11. https://doi.org/10.1186/1471-2172-15-11

11. Alper MM, Garner PR (1985) Premature ovarian failure: its relationship to autoimmune disease. Obstet Gynecol Clin North Am 66(1):27-30

12. Conway GS, Kaltsas G, Patel A, Davies MC, Jacobs HS (1996) Characterization of idiopathic premature ovarian failure. Fertil Steril 65(2):337-341. https:// doi.org/10.1016/s0015-0282(16)58095-9

13. Lebovic D, Rajesh N (2004) Premature ovarian failure: Think "autoimmune disorder". Sex Reprod Menopause 2(4):230-233

14. Iseme RA, McEvoy M, Kelly B, Agnew L, Walker FR, Attia J (2017) Is osteoporosis an autoimmune mediated disorder? Bone Rep 7:121-131. https:// doi.org/10.1016/j.bonr.2017.10.003
15. Mendoza-Pinto C, Rojas-Villarraga A, Molano-Gonzalez N, Jimenez-Herrera EA, Leon-Vazquez $M L$, Montiel-Jarquin A, Garcia-Carrasco M, Cervera R (2018) Bone mineral density and vertebral fractures in patients with systemic lupus erythematosus: A systematic review and meta-regression. PLoS ONE 13(6):e196113. https://doi.org/10.1371/ journal.pone. 0196113

16. Polovina SP, Miljic D, Zivojinovic S, Milic N, Micic D, Brkic VP (2017) The impact of thyroid autoimmunity (TPOAb) on bone density and fracture risk in postmenopausal women. Hormones 16(1):54-61. https://doi.org/10.14310/horm.2002.1719

17. Schmidt T, Schmidt C, Strahl A, Mussawy $H$, Rolvien T, JandI NM, Casar C, Oheim R, Schinke T, Lohse AW, Amling M, Schramm C, Barvencik F (2020) A system to determine risk of osteoporosis in patients with autoimmune hepatitis. Clin Gastroenterol Hepatol 18(1):226-233.e223. https:// doi.org/10.1016/j.cgh.2019.05.043

Hinweis des Verlags. Der Verlag bleibt in Hinblick auf geografische Zuordnungen und Gebietsbezeichnungen in veröffentlichten Karten und Institutsadressen neutral. 